\title{
ICT Effects and Enhancing Risk Management Information System (RMIS) in Medical Sector in Vietnam
}

\begin{abstract}
Measuring risk in medical sector in order to propose recommendation s is among concerns of scientists in recent years. In specific, using quantitative and analytical methods, author analyze ICT effects and IT applications in medical sector an hospitals, as well as analyze risk information to propose solutions to enhance risk management information system for Vietnam medical sector. Research findings show us that in order to give suitable suggestions for policy implications, firstly, study recognized that lower risks shown by beta values (<1) among 57\% of listed firms with lower risk. Last but not least, study stated that technology applications in medical sector can help doctors and nurses to manage, store and access better data and documents of patients. And Vietnam hospitals need to enhance more Risk management information system with strong support from IT (information technology) so that we could constantly improve service quality patient services, as well as build a favorable working environment for staff both professionally and professionally, etc.
\end{abstract}

